\title{
Endoscopic investigation in non-iron deficiency anemia: a cost to the health system without patient benefit
}

Authors

Institutions
Tamara Mogilevski', Rebecca Smith ${ }^{1}$, Douglas Johnson ${ }^{2,3}$, Patrick G. P. Charles ${ }^{2,4}$, Leonid Churilov ${ }^{5}$, Rhys Vaughan ${ }^{1,4}$, Ronald $\mathrm{Ma}^{6}$, Adam Testro ${ }^{1}$

Institutions are listed at the end of article. submitted 4. August 2015 accepted after revision 4. November 2015

\section{Bibliography}

DOI http://dx.doi.org/

10.1055/s-0041-109768

Published online: 15.1.2016

Endoscopy International Open 2016; 04: E198-E201

(c) Georg Thieme Verlag KG Stuttgart · New York

E-ISSN 2196-9736

\section{Corresponding author}

Tamara Mogilevski, MD

Department of

Gastroenterology

Austin Health, Heidelberg

Melbourne

Australia 3084

t.mogilevski@gmail.com
Background and aims: The indication for endoscopy to investigate anemia of causes other than iron deficiency is not clear. Increasing numbers of endoscopic procedures for anemia raises concerns about costs to the health system, waiting times, and patient safety. The primary aim of this study was to determine the diagnostic yield of endoscopy in patients referred to undergo investigation for anemia. Secondary aims were to identify additional factors enabling the risk stratification of those likely to benefit from endoscopic investigation, and to undertake a cost analysis of performing endoscopy in this group of patients. Methods: We performed a retrospective review of endoscopy referrals for the investigation of anemia over a 12-month period at a single center. The patients were divided into three groups: those who had true iron deficiency anemia (IDA), tissue iron deficiency without anemia (TIDWA), or anemia of other cause (AOC). Outcome measures included finding a lesion responsible for the anemia and a significant change of management

\section{Introduction}

$\nabla$

Anemia, defined as a reduction of the hemoglobin concentration, hematocrit, or red blood cell count, is an extremely common disorder affecting 1.62 billion people worldwide, or nearly onequarter of the world's population. Iron deficiency anemia (IDA) accounts for approximately one-half of this disease burden. The most common cause of IDA in developed countries is blood loss. This can be either overt or occult [1]. Upper and lower gastrointestinal endoscopy for the investigation of anemia is therefore common practice in public hospitals throughout Australia.

There is a significant body of literature justifying endoscopy in the setting of IDA [2]. The indication for endoscopy to investigate anemia of causes other than iron deficiency is less clear. Nonetheless, referral to exclude occult gastrointestinal as a result of endoscopy. A costing analysis was performed with an activity-based costing method.

Results: We identified 283 patients who underwent endoscopy to investigate anemia. A likely cause of anemia was found in 31 of 150 patients with IDA ( $21 \%)$ and 0 patients in the other categories $(P<0.001)$. A change of management was observed in 35 patients with IDA (23\%), 1 of 14 patients with TIDWA (7.14\%), and 8 of 119 patients with AOC $(6.7 \%)(P<0.001)$. The cost of a single colonoscopy or gastroscopy was approximated to be $\$ 2209$.

Conclusions: Endoscopic investigation for nonIDA comes at a significant cost to our institution, equating to a minimum of $\$ 293797$ per annum in extra costs, and does not result in a change of management in the majority of patients. No additional factors could be established to identify patients who might be more likely to benefit from endoscopic investigation. The endoscopic investigation of non-IDA should be minimized.

bleeding in patients with anemia of all causes remains a common occurrence [3]. Endoscopic investigation in this setting places further pressure on already strained hospital systems, with escalating costs, increased waiting times for outpatient procedures, and higher numbers of patients needing admission for bowel preparation.

Given these issues, a way of clearly stratifying the patients most likely to benefit from endoscopic investigation vs. those who are not is required. We hypothesize that the endoscopic investigation of non-IDA has less diagnostic utility and leads to less changes in management than does the endoscopic investigation of IDA.

In this study, our primary aim was to determine the diagnostic yield of endoscopy in patients referred to undergo investigation for anemia by comparing outcomes in those with true IDA vs. outcomes in those with other types of anemia. 
Our secondary aim was to identify and evaluate additional factors enabling the risk stratification of patients likely to benefit from endoscopic investigation. In addition, we analyzed the cost associated with endoscopic investigation for anemia within our institution.

\section{Methods}

$\nabla$

We performed a retrospective analysis of all patients referred for endoscopy with the indications of "anemia" and "IDA" at a major tertiary hospital in Melbourne, Australia, over a 12-month period. Ethics approval was granted by the hospital's human research ethics committee. Patients with acute overt bleeding, positive fecal occult blood test results, and known varices were excluded. Data were collected on patient demographics, referring unit, stated indication for referral, inpatient or outpatient procedure, type of endoscopy performed, hemoglobin level, and mean cell volume (MCV) at the time of referral. The Charlson Comorbidity Index was calculated with methods previously described in the literature [4]. Patients were separated into three categories: (i) true or early IDA (low hemoglobin level, low or normal MCV, low ferritin); (ii) tissue iron deficiency without anemia (TIDWA; normal hemoglobin, low ferritin, low or normal MCV); and (iii) anemia of other cause (AOC; low hemoglobin, normal ferritin, normal or high MCV). Outcome measures included the detection of a lesion on endoscopy explaining the anemia (defined as a gastrointestinal malignancy, bleeding peptic ulcer, active/luminal bleeding of any other cause, or celiac disease) and a significant change in management as a result of the endoscopy (defined as the removal of a polyp $\geq 10 \mathrm{~mm}$ in size, the removal of multiple polyps, argon photocoagulation treatment of nonbleeding angioectasia, or other change in management deemed significant by the clinician). Per hospital endoscopic protocol, when required, gastric and duodenal biopsy specimens were obtained to rule out celiac disease, pernicious anemia, and Helicobacter pylori infection, even when the endoscopic appearance was normal. If a patient had two separate pathologic conditions, the one most likely to result in anemia was taken as the reference.

To establish the cost associated with endoscopic procedures within our institution, an activity-based costing method that is widely used within hospitals in Victoria, Australia, was used [5]. This method allocates costs directly to individual patient episodes using various cost drivers, such as transaction, duration, and intensity cost drivers (e.g., theater duration), to obtain accurate costs. An up-to-date general ledger was extracted from the finance system and loaded into health care-specific clinical costing software to generate the cost of the admission being analyzed. The cost of patient care was divided into the cost of direct patient care and other, remaining cost centers not providing direct patient care, known as "overhead areas" or indirect costs. The total cost was then calculated from an addition of direct and indirect costs.

Descriptive statistics used included median and interquartile range (IQR; 25th and 75th percentiles). Univariable analyses for continuous variables were carried out using the Kruskal-Wallis one-way analysis of variance test. Binary variable analyses were performed with Fisher's exact test. A multiple logistic regression analysis was performed with the Charlson Comorbidity Index, inpatient vs. outpatient procedure, and type of endoscopy as independent variables and a referral for endoscopic investigation of $\mathrm{AOC}$ as the dependent variable. For all statistical tests, a two-

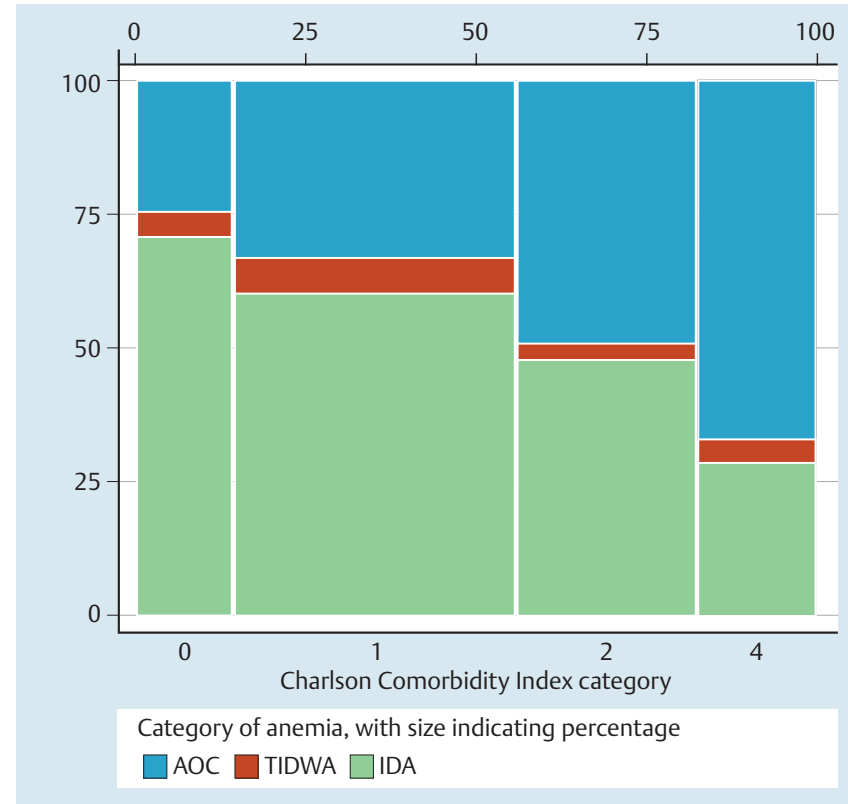

Fig. 1 Charlson Comorbidity Index and anemia by category. AOC, anemia of other cause; TIDWA, tissue iron deficiency without anemia; IDA, iron deficiency anemia.

tailed $P$ value of less than 0.05 was regarded as indicative of statistical significance. Stata/IC Statistical Software Release 13 (StataCorp LP; College Station, Texas, USA) was used for analysis.

\section{Results \\ $\nabla$}

In total, 283 patients with complete data were identified. Of these, 150 (53\%) met the criteria for IDA, 14 (4.9\%) for TIDWA, and $119(42 \%)$ for AOC. No significant differences were found for the patients' demographics, referring units, or types of procedure. Patients with a higher Charlson Comorbidity Index were significantly more likely to be referred for the investigation of non-IDA ( $\bullet$ Fig. 1), and patients with AOC were more likely to undergo an inpatient rather than an outpatient investigation ( Table 1). Specific lesions identified during endoscopy are listed in Table 2. A likely cause of anemia was found in 31 patients with IDA $(21 \%)$ and 0 patients in the other two categories $(P<$ $0.001)$. A change of management was observed in 35 of 150 patients with IDA (23\%), 1 of 14 patients with TIDWA, and 8 of 119 patients $(6.7 \%)$ with AOC $(P<0.001)$. A logistic regression analysis revealed that after adjustment for other factors, patients with AOC were significantly less likely to have a change of management than patients in with IDA (odds ratio $[\mathrm{OR}]=0.2$, confidence interval $[\mathrm{CI}] 0.08-0.0 .47, P<0.001)$.

The current costing of endoscopy procedures at our hospital includes $\$ 1585$ in direct costs, which include costs for direct medical care (e.g., medical and nursing staff labor), and \$625 in indirect costs, which include costs for administration, electricity, and information technology. Therefore, the total cost for either a gastroscopy or colonoscopy at our institution was approximated to be $\$ 2210$. 
Table 1 Baseline characteristics of 283 patients enrolled in a study of endoscopic investigation for non-iron deficiency anemia.

\begin{tabular}{|c|c|c|c|c|}
\hline Variable & IDA, $n=150$ (53\%) & TIDWA, $n=14$ (4.9\%) & $A O C, n=119(42.1 \%)$ & $P$ value \\
\hline Age, median (IQR), years & $72(54-79.25)$ & $69.5(57.25-77)$ & $70(57-78.5)$ & 0.9 \\
\hline Sex & & & & 0.186 \\
\hline Male, n (\%) & $71(47.3)$ & $7(50)$ & $68(57.1)$ & \\
\hline Female, n (\%) & $79(52.7)$ & $7(50)$ & $51(42.9)$ & \\
\hline Referring unit & & & & 0.148 \\
\hline General medicine, $\mathrm{n}(\%)$ & $38(25.3)$ & $0(0)$ & $30(25.2)$ & \\
\hline Gastroenterology, n (\%) & $55(36.7)$ & $8(57.1)$ & $29(24.4)$ & \\
\hline Surgery, n (\%) & $11(7.3)$ & $1(7.1)$ & $13(10.9)$ & \\
\hline General practitioner, n (\%) & $12(8)$ & $1(7.1)$ & $5(4.2)$ & \\
\hline Other, n (\%) & $34(22.7)$ & $4(28.6)$ & $42(35.3)$ & \\
\hline Inpatient procedure, n (\%) & $54(36)$ & $1(7.1)$ & $62(52.1)$ & 0.001 \\
\hline Procedure & & & & 0.294 \\
\hline Gastroscopy & $60(40)$ & $3(21.4)$ & $50(42)$ & \\
\hline Colonoscopy & $32(21.3)$ & $3(21.4)$ & $32(27)$ & \\
\hline Both & $58(38.7)$ & $8(57.1)$ & $37(31)$ & \\
\hline Categorical CCl & & & & 0.001 \\
\hline $0, n(\%)$ & $29(19.3)$ & $2(14.3)$ & $10(8.4)$ & \\
\hline $1, n(\%)$ & $71(47.3)$ & $8(57)$ & $39(32.8)$ & \\
\hline $2, n(\%)$ & $36(24)$ & $2(14.3)$ & $37(31.1)$ & \\
\hline $3, n(\%)$ & $14(9.3)$ & $2(14.3)$ & $33(27.7)$ & \\
\hline
\end{tabular}

IDA, iron deficiency anemia; TIDWA, tissue iron deficiency without anemia; AOC, anemia of other cause; CCI, Charlson Comorbidity Index.

Table 2 Characteristics of 45 lesions found on endoscopy.

\begin{tabular}{|lccll}
\hline Type of lesion & All lesions & IDA & TIDWA & AOC \\
\hline Malignancy, $\mathrm{n}(\%)$ & $13(29)$ & $13(100)$ & 0 & 0 \\
\hline Ulcer, $\mathrm{n}(\%)$ & $7(16)$ & $7(100)$ & 0 & 0 \\
\hline Active bleeding, $\mathrm{n}(\%)^{1}$ & $10(22)$ & $10(100)$ & 0 & 0 \\
\hline Polyps, $\mathrm{n}(\%)^{2}$ & $14(31)$ & $5(36)$ & $1(7)$ & $8(57)$ \\
\hline Nonbleeding angioectasia requiring argon plasma coagulation, $\mathrm{n}(\%)$ & 0 & 0 & 0 & 0 \\
\hline Other, $\mathrm{n}(\%)^{3}$ & $1(2)$ & $1(100)$ & 0 & 0 \\
\hline
\end{tabular}

IDA, iron deficiency anemia; TIDWA, tissue iron deficiency without anemia; AOC, anemia of other cause.

${ }^{1}$ Including blood in lumen, bleeding angioectasia, bleeding erosive gastropathy, and bleeding vessels.

2 Removal of polyp $10 \mathrm{~mm}$ or larger in size or removal of multiple polyps.

${ }^{3}$ One case of small-bowel adenocarcinoma found on capsule endoscopy after negative findings on colonoscopy and gastroscopy.

\section{Discussion}

$\nabla$

Our study has shown that a significant proportion of patients are referred to our hospital for the endoscopic investigation of nonIDA. Interestingly, referrals for non-IDA increased as the Charlson Comorbidity Index rose, perhaps reflecting the large number of investigations performed in this group of patients. Within our cohort, no patients with anemia of a cause other than iron deficiency obtained an explanation for their anemia after endoscopic investigation, and a significantly smaller proportion of these patients than of those with IDA had a change in management as a result of endoscopy. We were unable to draw conclusions from the cohort of patients within the TIDWA subgroup because of the small sample size and the fact that this cohort likely represents a group of patients with early IDA. No other factors were identified that could lead to better stratification in the referral of patients for endoscopy.

This study highlights the fact that in the absence of iron deficiency, the chance of finding significant pathology on endoscopy to explain anemia is likely to be small. Only 8 of the 119 patients referred for the investigation of AOC had a change in management as a result of endoscopy. We believe that these findings are consistent with the background incidence of the conditions that required treatment - for example, colonic polyps - and therefore are not likely to justify endoscopic investigation in this group of patients.

There is minimal information in the literature regarding endoscopic evaluation in patients with non-IDA. One such retrospective analysis looked at 100 consecutive patients who were investigated endoscopically for non-IDA. The findings were similar to ours in that the prevalence of lesions found to be responsible for anemia was significantly lower in the non-IDA group than in the IDA group ( $8 \%$ vs. $22.9 \%, P<0.001$ ). Interestingly, the patients who had non-IDA were also more likely to have an alternative cause of their anemia identified later, with chronic kidney disease and hematological disease among the most common causes [3]. These conclusions are reflected in clinical practice guidelines, which suggest that endoscopic investigation is likely to result in little diagnostic yield in patients who have iron deficiency without anemia, and that if performed, endoscopic investigation should be limited to those older than 50 years of age, in whom routine colon cancer screening is recommended [6].

The fact that the referring unit, procedure performed, and Charlson Comorbidity Index were not significantly associated with specified outcomes likely reflects otherwise appropriate referrals within our institution. Previous attempts to identify factors contributing to a higher likelihood of finding a lesion responsible for IDA have shown that only older age, male gender, and lower MCV 
are predictive of finding a lesion responsible for IDA [7,8]. The rate of lesion detection in our study may be somewhat lower than previously published rates [9-11]. This discrepancy may be explained by other studies' use of inclusion criteria that accepted patients with symptoms and positive fecal occult blood test results, as well as their use of less stringent criteria for what was defined as a lesion responsible for anemia (e.g., esophagitis or gastritis).

We have found that endoscopic investigation for non-IDA comes at a significant cost to our institution. If the patients without IDA did not undergo endoscopy, the savings for our institution would equate to approximately $\$ 293797$ per annum. This value is even higher when patients who require hospital admission for bowel preparation are taken into account, with the average cost of a day in the hospital on a general medical ward being approximately $\$ 720$.

There were several limitations to our study. First, the retrospective nature of the study inherently introduces confounders. One potential source of confounding was that patients may have received undocumented iron supplementation. Second, in order to clearly delineate what was a significant "cause for anemia" and "change in management," strict parameters had to be used for the definition. It is possible that a minority of patients had an endoscopic lesion associated with anemia that was not accounted for in our criteria.

In conclusion, a large proportion of patients were referred for the endoscopic investigation of non-IDA within our cohort. Patients with a higher Charlson Comorbidity Index were more likely than those with a lower score to be referred for endoscopic investigation in the context of non-IDA. Endoscopic investigation for nonIDA comes at a significant cost to our institution and has provided minimal yield both in finding the cause of the patients' anemia and in altering their management. Referring unit, procedure performed, and Charlson Comorbidity Index were not associated with specified outcomes. We believe that the wide referral base within our hospital makes these results generalizable to other organizations.

Given the escalating costs of our health system, finding ways to minimize spending without compromising patient care is of paramount importance. The endoscopic investigation of patients with non-IDA is one such area. Endoscopic investigation in these patients should be minimized because it rarely yields useful information or results in changes to patient management. Further investigation in this field should focus on determining the causes of anemia in the cohort of patients with non-IDA in order to help guide clinicians toward investigations that are more likely to yield results.

\section{Competing interests: None}

Institutions

${ }^{1}$ Department of Gastroenterology, Austin Health, Heidelberg, Melbourne, Australia

${ }^{2}$ Department of Infectious Diseases, Austin Health, Heidelberg, Melbourne, Australia

${ }^{3}$ Department of General Medicine, Austin Health, Heidelberg, Melbourne, Australia

${ }^{4}$ The University of Melbourne (Austin Health), Melbourne, Victoria, Australia

${ }^{5}$ The Florey Institute of Neuroscience and Mental Health, Melbourne, Victoria, Australia

${ }^{6}$ Department of Clinical Costing, Austin Health, Heidelberg, Melbourne, Australia

\section{References}

1 de Benoist B, McLean E, Egli I, Cogswell M. Worldwide prevalence of anaemia 1993-2005. WHO global database on anaemia. Geneva, Switzerland: World Health Organization; 2008: Available from: http:// apps.who.int/iris/bitstream/10665/43894/1/9789241596657_eng.pdf (Accessed 6 November 2015)

2 Andrews J, Sheppard S, Strasser S. Iron deficiency. Gastroenterological Society of Australia. Mulgrave, Victoria, Australia: Digestive Health Foundation; 2011: Available from: http://doctormel.com.au/wp-content/uploads/2012/03/Iron_def.pdf (Accessed 6 November 2011)

3 Powell N, McNair A. Gastrointestinal evaluation of anaemic patients without evidence of iron deficiency. Eur J Gastroenterol Hepatol 2008; 20: $1094-1100$

4 Charlson M, Szatrowski TP, Peterson J et al. Validation of a combined comorbidity index. J Clin Epidemiol 1994; 47: 1245-1251

5 Cooper R, Kaplan RS. Measure costs right: make the right decisions. Harvard Business Review 1988: (September-October) 96-103

6 Goddard AF, McIntyre AS, Scott BB. Guidelines for the management of iron deficiency anaemia. Gut 2000; 46: Suppl. 3-4, IV1 - IV5

7 Capurso G, Baccini F, Osborn J et al. Can patient characteristics predict the outcome of endoscopic evaluation of iron deficiency anemia: a multiple logistic regression analysis. Gastrointest Endosc 2004; 59: $766-771$

8 Majid S, Salih M, Wasaya $R$ et al. Predictors of gastrointestinal lesions on endoscopy in iron deficiency anemia without gastrointestinal symptoms. BMC Gastroenterol 2008; 8: 52

9 McIntyre AS, Long RG. Prospective survey of investigations in outpatients referred with iron deficiency anaemia. Gut 1993; 34: 1102 - 1107

10 Bampton PA, Holloway RH. A prospective study of the gastroenterological causes of iron deficiency anaemia in a general hospital. Aust N Z J Med 1996; 26: $793-799$

11 Rockey DC, Cello JP. Evaluation of the gastrointestinal tract in patients with iron-deficiency anemia. N Engl J Med 1993; 329: 1691 - 1695 